\title{
Corpus callosum index and long-term disability in multiple sclerosis patients
}

\author{
Özgür Yaldizli • Ramin Atefy $\cdot$ Achim Gass $\cdot$ Dietrich Sturm • \\ Stephanie Glassl $\cdot$ Barbara Tettenborn • Norman Putzki
}

Received: 30 September 2009/Revised: 4 February 2010/ Accepted: 8 February 2010/Published online: 3 March 2010

(c) Springer-Verlag 2010

\begin{abstract}
Prediction of long-term disability in patients with multiple sclerosis (MS) is essential. Magnetic resonance imaging (MRI) measurement of brain volume may be of predictive value but sophisticated MRI techniques are often inaccessible in clinical practice. The corpus callosum index (CCI) is a normalized measurement that reflects changes of brain volume. We investigated medical records and 533 MRI scans at diagnosis and during clinical followup of $169 \mathrm{MS}$ patients (mean age $42 \pm 11$ years, $86 \%$ relapsing-remitting MS, time since first relapse $11 \pm$ 9 years). CCI at diagnosis was $0.345 \pm 0.04$ and correlated with duration of disease $(p=0.002 ; r=-0.234)$ and expanded disability status scale (EDSS) score at diagnosis $(r=-0.428 ; \quad p<0.001)$. Linear regression analyses identified age, duration of disease, relapse rate and EDSS at diagnosis as independent predictors for disability after mean of 7.1 years (Nagelkerkes' R:0.56). Annual CCI decrease was $0.01 \pm 0.02$ (annual tissue loss: $1.3 \%$ ). In secondary progressive MS patients, CCI decrease was double compared to that in relapsing-remitting MS patients $(p=0.04)$. There was a trend of greater CCI decrease in untreated patients compared to those who received disease modifying drugs $(p=0.2)$. CCI is an easy to use MRI
\end{abstract}

Ö. Yaldizli $(\bowtie)$

Department of Neurology, University of Basel,

Petersgraben 4, 4031 Basel, Switzerland

e-mail: OYaldizli@uhbs.ch

Ö. Yaldizli · R. Atefy · D. Sturm · S. Glassl · B. Tettenborn ·

N. Putzki

Department of Neurology, Cantonal Hospital St. Gallen,

St. Gallen, Switzerland

A. Gass

Department of Neurology/Neuroradiology, University of Basel, Basel, Switzerland marker for estimating brain atrophy in patients with MS. Brain atrophy as measured with CCI was associated with disability progression but it was not an independent predictor of long-term disability.

Keywords Multiple sclerosis · MRI - Atrophy · Corpus callosum - Disability $\cdot$ Disease modifying treatment

\section{Introduction}

Prediction of long-term disability in patients with multiple sclerosis (MS) is important for both patients and physicians. Several studies have been conducted to identify clinical characteristics such as relapse rate, gender or age in an attempt to predict long-term disability, although correlations were only modest $[6,36,42]$. Despite initial disease course (i. e. relapse rate) and early disability evolution (as measured by the expanded disability status scale, or EDSS) being of some prognostic value, long-term progression remains largely unpredictable. This uncertainty stresses the need for reliable para-clinical parameters such as magnetic resonance imaging (MRI). MRI techniques play an important role and can depict subclinical disease progression. The most established MRI parameters used in evaluating disease outcome and routinely used in clinical trials are hyperintensities on $\mathrm{T} 2$ weighted images, hypo-intensities in $\mathrm{T} 1$ weighted images and contrast enhancing lesions [22, 33, 48, 50]. However, several studies have revealed only a weak correlation between $\mathrm{T} 2$ lesion load and clinical disability [14, 16, 17, 35, 37]. New MRI techniques have been developed that offer the prospect of greater specificity including measurements of brain volume $[12,28,30]$. Sophisticated MRI techniques are laborious, need special software and are often inaccessible in clinical practice. In 
search of more practical bedside parameters the corpus callosum index (CCI) has been suggested as a marker for brain atrophy in MS patients [11]. Demonstrated correlation of CCI and atrophy has been measured with brain parenchymal fraction. In addition, CCI correlates with cognitive impairment in relapsing-remitting MS (RRMS) and secondary progressive MS (SPMS) patients. The corpus callosum is the largest compact white matter fiber bundle of the brain connecting both hemispheres [23]. Previous MRI and histopathological studies have consistently revealed the corpus callosum as one of the sites most commonly affected by demyelination and axonal loss $[2,10,20,32,38,45]$. The aim of this study was to investigate the relationship between $\mathrm{CCI}$ and long-term disability evolution in MS.

\section{Patients and methods}

\section{Study design}

This study is a cohort study of RRMS and SPMS patients based on an analysis of medical records and on serial MRI examinations from which a linear measurement (CCI) was retrospectively calculated.

\section{Patients}

From October 2008 to April 2009, we screened all patients attending our MS outpatient clinic. Inclusion criteria were patients between 18 and 60 years at presentation, diagnosis of relapsing-remitting MS (RRMS) according to revised McDonald criteria 2005 [39] or secondary progressive MS (SPMS) [31] and availability of at least one MRI examination performed in our radiological institute. Exclusion criteria were any brain pathology other than MS, pure spinal manifestation of demyelination, neuromyelitis optica and primary progressive MS (PPMS). We excluded PPMS patients because only a limited number were followed at our center. Clinical characteristics were derived from medical records. Disease duration was defined as time since first manifestation of disease. Relapses were defined as the development of new or recurrent neurological symptoms not associated with fever or infection lasting at least $24 \mathrm{~h}$ and confirmed by a Swiss or German board-certified neurologist. Secondary progression was defined as continued deterioration of disability for at least 12 months with or without interposed relapses after an initial relapsingremitting course of disease [31]. Patients were not part of a clinical trial. Most patients were treated with disease modifying drugs. The reasons for patients being untreated or untreated during certain periods were due to previous side effects or a personal decision. Patients were regarded as untreated if cumulative treatment duration was $<3$ months.
MRI analysis

All MRI scans were performed in our radiological institute (1.5 Tesla Sigma Magnetom Scanner, Siemens AG, Germany) and included the following: an axial pre and post gadolinium $\mathrm{T} 1$ weighted, sagittal $\mathrm{T} 1$ weighted and fluid attenuated inversion recovery sequence (FLAIR), axial T2 weighted and axial FLAIR sequences. All MRI scans were analyzed by the same examiner (OY). For reliability analysis, CCI of 266 MRI scans were measured independently by two additional investigators (RA, SG). All raters were blind to patient clinical data at assessment. The concordance rate among the three investigators was 0.93 (Cronbach alpha). Both T2 and T1 lesion load and contrast enhancement were determined by Swiss board-certified radiologists. T2 lesion load was dichotomized as either $<9$ $\mathrm{T} 2$ lesions or $\geq 9 \mathrm{~T} 2$ lesions. $\mathrm{T} 1$ lesions and contrast enhancing lesions were categorized as present or absent. CCI was obtained on conventional best mid-sagittal T1 weighted images by drawing a line at the greatest anteroposterior diameter of the corpus callosum and a perpendicular line at its midpoint (Fig. 1). T1 weighted images are chosen because of the higher signal and contrast to noise ratio [11]. The measurements have been performed on a picture archiving and communication system (PACS) workstation and if unavailable directly on films. The best mid-sagittal image was found by identification of the middle part of the fornix. Anterior, posterior and medium segments of the corpus callosum were measured and normalized to its greatest antero-posterior diameter with an electronic calliper or by computerized measurement tool on screen. The concordance rate of electronic calliper and on-screen measurement was 0.97 based on 189 MRI scans from 71 patients. Higher CCI values indicate a higher corpus callosum volume.

\section{Statistical analysis}

Demographic data are presented as mean \pm standard deviation. For comparison of continued variables we used the Mann-Whitney test for independent samples. Reliability was assessed as Cronbach alpha. Correlation analyses including CCI, age, disease duration, MRI parameters and EDSS were performed with the non-parametric rank correlation analysis Spearman-Rho and an adjusted significance level of 0.005 ( $p=0.05 / 10$; Bonferroni correction). A backward directed linear regression analysis was performed to identify best predictors for disability status (last EDSS). Last EDSS was used as an outcome measure (continued variable). The potential predictors were CCI at diagnosis (continued variable), age (continued variable), duration of disease (continued variable), total number of relapses (continued variable), total number of steroid 

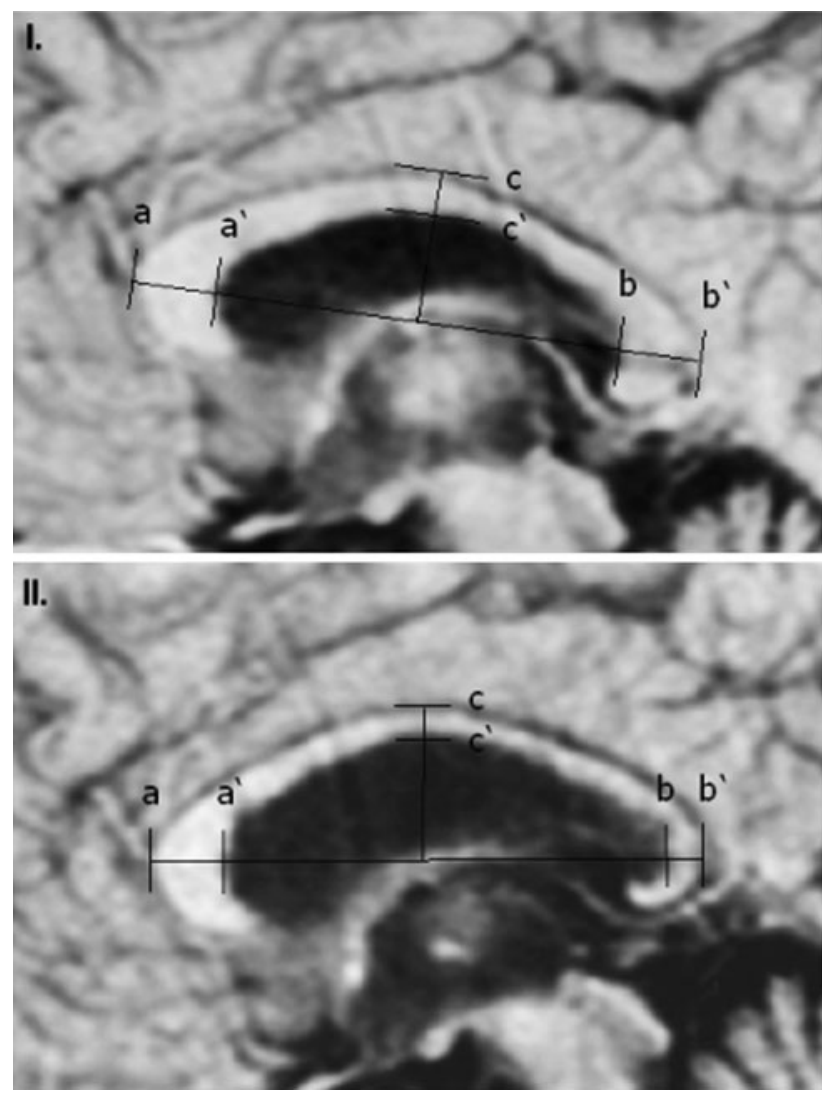

Fig. 1 MRI of a 31 year old male patient with first diagnosis of RRMS (Fig. 1; EDSS 1.5; CCI $=0.321$ ) and follow-up 4 years later (Fig. 2; EDSS 4.0; $\mathrm{CCI}=0.259$ ). $\mathrm{CCI}$ is calculated as $\left(a a^{\prime}+b b^{\prime}+c c^{\prime}\right) / a b^{\prime}$. Lower CCI values indicate a lower corpus callosum volume. CCI decrease of $20 \%$ in 4 years was associated with an increase of EDSS from 1.5 to 4.0. (CCI corpus callosum index EDSS expanded disability status scale, RRMS relapsing-remitting multiple sclerosis)

pulses (continued variable), T2 lesion load (categorical variable: $<9 / \geq 9$ ), gadolinium enhancement (categorical variable: yes/no), EDSS at diagnosis (continued variable) and course of disease (categorical variable: RRMS/SPMS). We used SPSS (Windows version 14; SPSS, Chicago, IL) for all statistical analysis.

\section{Results}

We screened 176 consecutive patients. Excluded from the analysis were one patient with hydrocephalus due to congenital aqueduct stenosis, one patient with concomitant idiopathic epilepsy and five patients with isolated spinal demyelination. Thus, this analysis comprised data of 169 MS patients. We investigated medical records and all available MRI scans $(n=533 ; 3.2 \pm 1.7$ MRIs per patient). Corresponding EDSS data (assessed within 2 months of MRI) were available at 388 time points. The
Table 1 Demographic data

\begin{tabular}{|c|c|}
\hline \multicolumn{2}{|l|}{ Patient characteristics } \\
\hline$n$ & 169 \\
\hline Age (years \pm SD) & $42.0 \pm 11.3$ \\
\hline \multicolumn{2}{|l|}{ Gender $(\%)$} \\
\hline Male & $43(25.4)$ \\
\hline Female & $126(74.6)$ \\
\hline \multicolumn{2}{|l|}{ Course of disease $(\%)$} \\
\hline RRMS & $1455.8)$ \\
\hline SPMS & $24(14.2)$ \\
\hline Time from first manifestation to diagnosis (years) & $3.9 \pm 3.0$ \\
\hline $\begin{array}{l}\text { Duration (years } \pm \text { SD) since first manifestation of } \\
\text { disease }\end{array}$ & $10.9 \pm 8.8$ \\
\hline EDSS at diagnosis & $2.6 \pm 0.7$ \\
\hline Last EDSS ${ }^{\mathrm{a}}$ & $2.97 \pm 0.86$ \\
\hline \multicolumn{2}{|l|}{$\mathrm{T} 2$ lesion load at diagnosis $(\%)$} \\
\hline$<9$ & $74(6.8)$ \\
\hline$\geq 9$ & $95(56.2)$ \\
\hline \multicolumn{2}{|l|}{$\mathrm{T} 1$ lesions at diagnosis $(\%)$} \\
\hline No & $99(58.6)$ \\
\hline Yes & $70(41.4)$ \\
\hline \multicolumn{2}{|l|}{ Contrast enhancement at diagnosis (\%) } \\
\hline No & $134(9.3)$ \\
\hline Yes & $23(13.6)$ \\
\hline No gadolinium applied & $12(7.1)$ \\
\hline Total number of relapses including first event & $4.2 \pm 1.8$ \\
\hline Total n/o steroid pulse therapies & $2.3 \pm 1.0$ \\
\hline \multicolumn{2}{|l|}{ Disease modifying therapy $(\%)$} \\
\hline Never & $22(13.0)$ \\
\hline Yes (at any time) & $147(87.0)$ \\
\hline Median treatment duration (range; years) & $\begin{array}{l}4.0(0.2- \\
23.75)\end{array}$ \\
\hline Currently on treatment & $118(69.8)$ \\
\hline
\end{tabular}

$R R M S$ relapsing-remitting multiple sclerosis, SPMS secondary progressive multiple sclerosis, EDSS expanded disability status scale

${ }^{\mathrm{a}}$ mean follow-up $7.1 \pm 6.4$ (median $5.2 ; 0.2-33.2$ years)

demographic characteristics of patients are given in Table 1 and the results from correlation analyses in Table 2.

$\mathrm{CCI}$ at diagnosis was $0.345 \pm 0.04$ and correlated with disease duration and EDSS at diagnosis. CCI at diagnosis did not correlate with age at presentation, age at first manifestation, gadolinium enhancing lesions at diagnosis, $\mathrm{T} 1$ or T2 lesion load at diagnosis or gender. EDSS at diagnosis was $2.6 \pm 0.7$ and correlated with course of disease, disease duration and T1 lesions $(p=0.002$; EDSS $2.1 \pm 1.2$ in the group without $\mathrm{T} 1$ lesions versus $3.05 \pm 1.7$ in the group with T1 lesions). EDSS at diagnosis did not correlate with gender, gadolinium enhancement at diagnosis, T2 lesion load $(<9 / \geq 9)$ or age at first 
Table 2 Correlation analyses of corpus callosum index (CCI) at diagnosis and expanded disability status scale (EDSS) at diagnosis and after mean of 7.1 years (last EDSS)

\begin{tabular}{|c|c|c|}
\hline \multicolumn{2}{|l|}{ Correlations } & \multirow{2}{*}{$\begin{array}{l}\text { Significance; } r \text { value } \\
p=0.002 ; r=-0.234\end{array}$} \\
\hline $\mathrm{CCI}$ at diagnosis versus & Duration of disease based on first manifestation & \\
\hline & EDSS at diagnosis & $p<0.001 ; r=-0.428$ \\
\hline & Age at time of the study & n.s. \\
\hline & Age at first manifestation & n.s. \\
\hline & Gd positive lesion at diagnosis & n.s. \\
\hline & T1 lesion at diagnosis & n.s. \\
\hline & $\mathrm{T} 2$ lesion at diagnosis & n.s. \\
\hline & Gender & n.s. \\
\hline \multirow[t]{7}{*}{ EDSS at diagnosis versus } & Course of disease (RRMS/SPMS) & $p=0.001$ \\
\hline & Disease duration since first manifestation of disease & $p<0.001 ; r=0.456$ \\
\hline & Presence of $\mathrm{T} 1$ lesion(s) at diagnosis & $p=0.002$ \\
\hline & Gender & n.s. \\
\hline & Gd positive lesions & n.s. \\
\hline & $\mathrm{T} 2$ lesion load $(<9 / \geq 9)$ & n.s. \\
\hline & Age at first manifestation & n.s. \\
\hline \multirow[t]{13}{*}{ Last EDSS versus } & $\mathrm{CCI}$ at diagnosis & $p=0.002 ; r=0.283$ \\
\hline & $\mathrm{CCI}$ at last MRI & $p<0.001 ; r=0.301$ \\
\hline & Age at time of study & $p<0.001 ; r=0.392$ \\
\hline & Duration of disease since first manifestation & $p<0.001 ; r=0.496$ \\
\hline & EDSS at diagnosis & $p<0.001 ; r=0.436$ \\
\hline & Number of relapses & $p<0.001 ; r=0.301$ \\
\hline & Number of steroid pulse therapies & $p<0.001 ; r=0.3$ \\
\hline & Course of disease (RRMS vs. SPMS) & $p<0.001$ \\
\hline & Age at first manifestation of disease & n.s. \\
\hline & T1 lesion load at diagnosis & n.s. \\
\hline & $\mathrm{T} 2$ lesion load at diagnosis & n.s. \\
\hline & Gd positive lesions at diagnosis & n.s. \\
\hline & Treatment with disease modifying drugs & n.s. \\
\hline
\end{tabular}

RRMS relapsing-remitting MS, SPMS secondary progressive MS, Gd Gadolinium, n.s. not significant

manifestation. After a mean time follow-up of $7.1 \pm$ 6.4 years (median 5.2 years; range $0.2-33.27$ years) CCI decreased from $0.345 \pm 0.04$ to $0.317 \pm 0.03$ by a mean of $0.01 \pm 0.02$ per year, whereas EDSS increased from $2.6 \pm 0.7$ to $2.97 \pm 0.86$ (last EDSS). EDSS worsened in 49 patients $(27.4 \%)$ by at least one point over the observational period. Last EDSS correlated with CCI at diagnosis, CCI at last MRI, age at time of study, disease duration, EDSS at diagnosis, number of relapses, number of steroid pulse therapies and course of disease (2.61 \pm 1.36 in the RRMS group vs. $5.07 \pm 2.15$ in the PPMS group; $p<0.001$ ). Last EDSS did not correlate with age at first manifestation of disease, T1 lesion load at diagnosis, $\mathrm{T} 2$ lesion load at diagnosis or gadolinium positive lesions at diagnosis or treatment with disease modifying drugs (DMD) (Table 2).

We conducted a linear regression analysis with last EDSS as a dependent variable and with CCI at diagnosis, age today, disease duration, total number of relapses, total number of steroid pulses, T2 lesion load at diagnosis, presence of gadolinium positive lesion, EDSS at diagnosis and course of disease as covariates. This analysis revealed EDSS at diagnosis, disease duration, age today and number of relapses as independent predictors for the last EDSS (Nagelkerkes' R: 0.56, Table 3).

Twenty-four patients (17 female, 7 male) had secondary progression after a mean duration of $6.2 \pm 4.6$ years. These patients had lower CCI values at diagnosis $(p=0.003)$ compared to those without secondary progression $(0.308 \pm 0.08$ in SPMS vs. $0.353 \pm 0.06$ in RRMS patients). In SPMS patients, annual CCI decrease was higher than in RRMS patients (CCI reduction in RMS patients $-0.006 \pm 0.015$ versus $-0.02 \pm 0.011 ; p=0.04$, Fig. 2). We saw in patients with $\mathrm{T} 2$ lesion load $\geq 9$, T1 hypo-intensities or contrast enhancing lesions at first examination a trend of greater and earlier CCI decrease, 
Table 3 Linear regression analysis using last expanded disability status scale (EDSS) as a dependent variable and age at first manifestation of disease, relapse rate, number of steroid pulses, T2 lesion load, T1 lesion load and corpus callosum index (CCI) as covariates

\begin{tabular}{|c|c|c|c|c|c|}
\hline & Coefficient & Standard error & Standardized coefficient beta & T-value & $p$ value \\
\hline Constant & -0.680 & 0.539 & & -1.261 & 0.211 \\
\hline Age & 0.039 & 0.013 & 0.257 & 3.057 & 0.003 \\
\hline Duration of disease & 0.108 & 0.028 & 0.383 & 3.787 & 0.000 \\
\hline Total number of relapses & 0.152 & 0.059 & 0.201 & 2.560 & 0.012 \\
\hline EDSS at diagnosis & 0.273 & 0.112 & 0.236 & 2.437 & 0.017 \\
\hline
\end{tabular}

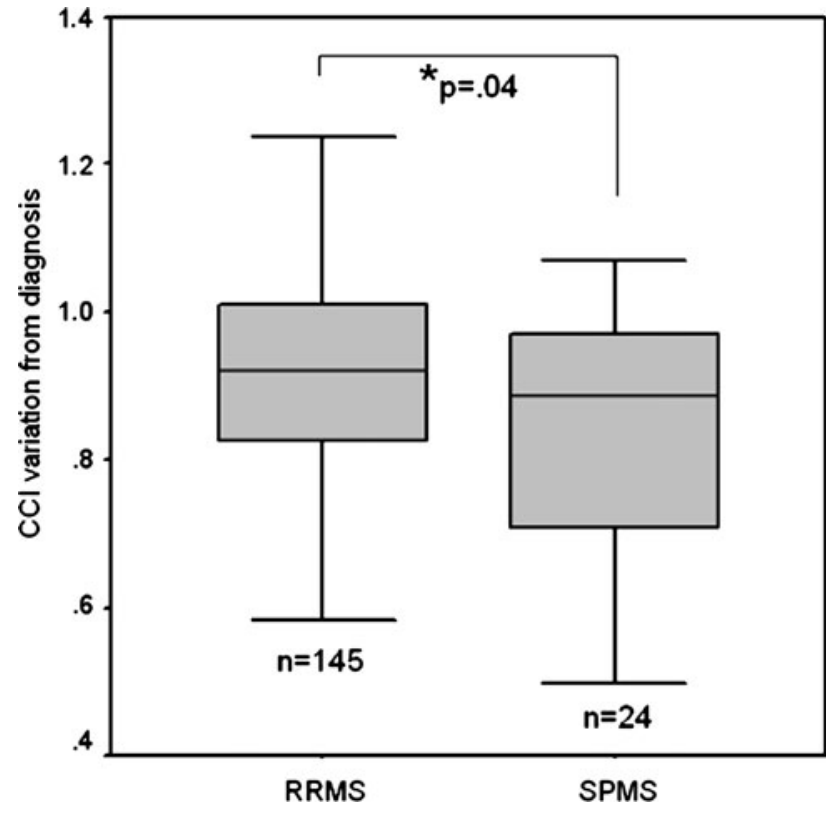

Fig. 2 Box-and-whisker diagram of the corpus callosum index (CCI) change after a mean time of 7 years from baseline (1.0), relapsingremitting multiple sclerosis (RRMS) versus secondary progressive multiple sclerosis (SPMS)

defined as time between first and second MRI after a mean of $2.5 \pm 1.5$ years $(p=0.07$; data not shown) without reaching significance.

Eighty-seven percent of patients $(n=147)$ received DMDs (mean treatment duration $3.98 \pm 0.15$ years (median 4.0 years, range $0.2-23.75$ years). Baseline characteristics of patients with and without DMD treatment are given in Table 4. Twenty-two patients were not treated with DMDs. They were more likely to have secondary progressive MS $(p=0.02)$, higher annualized relapse rate and more common gadolinium positive lesions on baseline MRI. Patients treated with and without DMDs did not differ in terms of sex, age, disease duration, $\mathrm{T} 2$ or $\mathrm{T} 1$ lesions at diagnosis, CCI or EDSS at diagnosis or last visit, number of relapses or number of steroid pulse therapies. Annual CCI decrease was three times higher in patients without DMD $(-0.0174 \pm 0.018 ; n=22)$ compared to patients who received DMD $(-0.005 \pm 0.014$ per year; $n=147 ; p=0.2$ ).

\section{Discussion}

Brain atrophy and long-term disability

The main finding of our study was that brain atrophy as measured by CCI was associated with disability progression in MS patients. However, CCI was not an independent predictor of long-term disability. Our analysis revealed that about $56 \%$ of the variance of future disability is predicted by age of the patient at presentation, duration of disease, number of relapses and EDSS at diagnosis of MS, the latter being the best predictor for future disability.

Our results for brain atrophy as measured with CCI are in line with other investigations using more sophisticated MRI methods for evaluation of brain atrophy such as brain parenchymal fraction (BPF). Several studies indicate a strong relationship between brain atrophy and EDSS [9, 27, 44] or disease duration [3, 18, 21, 27, 43, 44]. A crosssectional study of 90 MS-patients [9] demonstrated that EDSS correlated with neo-cortical volume both in 65 RRMS and 25 PPMS patients $(r=-0.27$ to $r=-0.64$, $p<0.05$ ). Another study [27] found in 33 MS patients a lower brain parenchymal fraction (BPF) compared with age-matched controls. BPF correlated with disease duration and EDSS $(r=-0.51, p<0.05)$ but not with the number of detectable MS lesions.

On the other hand, several studies have failed to find a significant correlation between brain atrophy and disability $[4,13,15,18,26,40,47]$. One such study could not find a correlation among EDSS, age and $\operatorname{BPF}(n=30)$ [18]. In another study, an assessment of whole brain, grey matter and white matter atrophy by quantitative MRI in $26 \mathrm{MS}$ patients found no correlations between EDSS and any lesion type or fractional tissue volume [4].

The authors have advanced different arguments to explain these contradictory results. Some authors have discussed EDSS as an inappropriate score to estimate a full range of impairment since cognitive dysfunction is 
Table 4 Patient and disease characteristics in patients exposed to DMD ( $\geq 3$ months) and untreated patients

\begin{tabular}{|c|c|c|c|}
\hline \multirow[t]{2}{*}{ Parameters } & \multicolumn{2}{|l|}{ DMD } & \multirow[t]{2}{*}{ Significance } \\
\hline & No & Yes & \\
\hline$n$ & 22 & 147 & \\
\hline \multicolumn{4}{|l|}{$\operatorname{Sex}(\%)$} \\
\hline Male & $4(18.2)$ & $39(26.5)$ & 0.40 \\
\hline Female & $18(81.8)$ & $108(73.5)$ & \\
\hline \multicolumn{4}{|l|}{ Age (years) } \\
\hline At time of study & $43.5 \pm 20.1$ & $41.1 \pm 12.3$ & 0.12 \\
\hline At first manifestation & $29.8 \pm 23.3$ & $30.6 \pm 12.4$ & 0.49 \\
\hline Disease duration (years) & $13.8 \pm 11.0$ & $10.4 \pm 8.3$ & 0.24 \\
\hline Course of disease & & & 0.02 \\
\hline RRMS & $15(68.2)$ & $130(88.4)$ & \\
\hline SPMS & $7(31.8)$ & $17(11.6)$ & \\
\hline T2 lesion load at diagnosis & & & 0.53 \\
\hline$<9$ & $11(50)$ & $63(42.9)$ & \\
\hline$\geq 9$ & $11(50)$ & $84(57.1)$ & \\
\hline T1 lesions at diagnosis & & & 0.61 \\
\hline No & $14(63.6)$ & $85(57.8)$ & \\
\hline Yes & $8(36.4)$ & $62(42.2)$ & \\
\hline Gd positive lesion at diagnosis ${ }^{\mathrm{a}}$ & & & 0.03 \\
\hline No & $20(100)$ & $114(3.2)$ & \\
\hline Yes & $0(0)$ & $23(16.8)$ & \\
\hline \multicolumn{4}{|l|}{ CCI } \\
\hline At diagnosis & $0.342 \pm 0.058$ & $0.347 \pm 0.069$ & 0.68 \\
\hline At last visit ${ }^{\mathrm{b}}$ & $0.306 \pm 0.067$ & $0.317 \pm 0.077$ & 0.51 \\
\hline \multicolumn{4}{|l|}{ EDSS } \\
\hline At diagnosis & $3.4 \pm 2.1$ & $2.4 \pm 1.3$ & 0.23 \\
\hline At last visit ${ }^{\mathrm{b}}$ & $3.6 \pm 2.3$ & $2.9 \pm 1.6$ & 0.25 \\
\hline Number of relapses ${ }^{c}$ & $2.85 \pm 1.8$ & $4.38 \pm 3.7$ & 0.13 \\
\hline Number of steroid pulse therapies & $1.23 \pm 1.1$ & $1.95 \pm 2.1$ & 0.30 \\
\hline Annualized relapse rate & $1.13 \pm 2.8$ & $0.87 \pm 1.3$ & 0.03 \\
\hline
\end{tabular}

$D M D$ disease modifying drug RRMS relapsing-remitting MS SPMS secondary progressive MS Gd Gadolinium $C C I$ corpus callosum index EDSS expanded disability status scale

${ }^{\text {a }}$ Not every patient received gadolinium (see Table 1)

b Last visit was after mean of 7.1 years

${ }^{c}$ Number of relapses since first demyelinating event

not adequately represented [13]. Significant negative correlations between upper cervical cord volume and EDSS have been found, suggesting that higher EDSS scores seem to correlate more with spinal than cerebral manifestation of disease [29]. Other studies included patients with only mild disability, restricting the potential for detecting meaningful correlations [4, 26, 47]. Studies have also investigated heterogeneous MS groups with a short follow-up period [15]. Our study and the time series follow-up data it generated over 7 years may have made it easier to detect correlations between atrophy and disability.
Lesion load and brain atrophy

We found a weak correlation among presence of $\mathrm{T} 1$ lesions, contrast enhancement and atrophy (CCI) while T2 lesion load did not correlate with CCI. The relationship between lesion load and brain atrophy is discussed controversially in the literature. One study found significant correlations with $\mathrm{r}$ values reaching -0.78 for $\mathrm{T} 2$ lesion volume and -0.59 for T1 lesion volume (RRMS only) [4]. Another study failed to find correlations between BPF and lesion load [3, 4, 19] but this included patients with both RRMS and SPMS as was the case in our study. A strong 
negative correlation between T2 lesion volume and baseline BPF of 138 RRMS patients has been previously shown, while a weak correlation was found between the change in BPF over 3 months and $\mathrm{T} 2$ lesion volume at baseline [21]. This suggested that lesion load is only a weak predictor of progressive brain atrophy, a finding similar to our results. In another study the baseline T2 lesion volume indicated more pronounced atrophy (BPF) but $\mathrm{T} 2$ lesion volume accounted for only $8.2 \%$ of the variance in progressive brain atrophy [41]. Some authors have reported that grey matter volume rather than white matter volume is correlated with total $\mathrm{T} 2$ or $\mathrm{T} 1$ lesion volume [4, 18, 21, 24, 40, 43, 46, 49]. This would also explain the weak correlation in our study.

\section{Corpus callosum atrophy in different courses of disease}

In our study, CCI decreased by approximately 9\% over approximately 7 years. This change reflects an annual tissue loss of $1.3 \%$ and is consistent with other studies using BPF method (0.5-1.3\% annual brain volume loss) $[1,5,8,21]$. These findings have been demonstrated elsewhere, underlining the remarkable correlation of the easy bedside test CCI and BPF [11]. CCI decrease was more than double in SPMS patients compared to RRMS patients. CCI reduction in SPMS patients was similar to results from a separate study [11]. Some studies have reported reduced whole brain volumes in patients with SPMS compared with age-matched RRMS patients [3, 19, $44,51]$. One such study measured white matter and grey matter atrophy using a fully automated multi-parametric segmentation method in 597 MS patients and found more atrophy of both white and grey matter in SPMS patients [46]. Another study reported that normalized brain volume was lower in 18 patients with SPMS $(1,406 \mathrm{ml})$ than in 36 RRMS patients $(1,473 \mathrm{ml})$ [51]. Researchers detected higher rates of brain parenchymal loss in 9 SPMS patients than in 27 RRMS patients $(-1.5 \%$ per year for RRMS vs. $-2.0 \%$ per year for SPMS) [19]. Other studies found the periventricular region sensitive to brain atrophy in SPMS patients. For example, in a cohort of 83 patients with MS, researchers analyzed the rate of brain volume loss over time using BPF as a measure of whole brain atrophy and normalized ventricular volume as a measure of central atrophy [26]. They reported that the annualized rate of brain parenchymal atrophy did not significantly differ between subtypes of patients $(0.7 \%$ per year), whereas the annualized rate of ventricular enlargement was higher for SPMS patients. This result was independently confirmed through an investigation of ventricular enlargement over 1 year [7]; ventricular enlargement in all MS patients over the 1 year period was reported, but significantly greater enlargement occurred in the SPMS group. A difference in the brain atrophy rate between subgroups of MS has also been found, with the largest decrease $(0.9 \%$ per year) in patients with SPMS and the smallest $(0.6 \%$ per year) in patients with PPMS [15]. These differences were very close to the rate reported by others [26].

Disease modifying treatment and brain atrophy

DMDs are the mainstays of long-term therapy of MS. They may decelerate brain volume loss by reducing inflammation and possibly promoting remyelination. Brain volume loss can occur in every stage of disease, but is probably more accentuated with longer disease duration and/or SPMS. Although brain atrophy as measured with CCI was three times higher in untreated patients, it did not reach statistical significance. Several confounders could impact this finding (e.g. disease type) and the low number of untreated patients makes it hard to produce definitive conclusions. It was demonstrated that DMDs may impact atrophy but findings for the different preparations were controversial [25, 41, 52].

Future studies should include either CCI or parameters of corpus callosum volumetry to define effects of DMDs on the parameter of brain atrophy.

\section{Limitations of our study}

The retrospective evaluation of the clinical course and the measurement of only white matter atrophy represent limitations of our study. Recent studies revealed that grey matter atrophy is associated with disability. Segmentation techniques have made it possible to distinguish between grey matter and white matter [34]. Further studies should combine linear measurement systems for grey and white matter. Our study population was representative of the population treated in our outpatient department (i. e. $86 \%$ RRMS and 14\% SPMS). We excluded patients with concomitant brain pathology and patients without brain lesions to enhance the reliability of our data. Most of our patients (87\%) were exposed to DMDs and had mild disability after 7 years. The strengths of our study were the long follow-up period and blinded MRI assessment. CCI was a reliable MRI measurement with an interrater reliability of more than $90 \%$, which is similar to previously published data [11]. EDSS assessment was performed by experienced neurologists who were certified for undertaking such assessments in clinical trials. Recent studies compared CCI with matched healthy controls [11]. Despite intersubject variations in the shape and size of corpus callosum including sex differences and handedness, our results show that CCI seems to be a robust parameter. 


\section{Conclusion}

Brain atrophy as measured by CCI is an important marker of subclinical disease course. CCI is easy to apply and correlates with long-term disability. When sophisticated MRI parameters of atrophy are not accessible, estimation of atrophy by CCI may be included either for follow-up evaluations alongside more established clinical (relapse rate, EDSS) and para-clinical assessments (presence of Gd lesions, T2 lesion load, presence of $\mathrm{T} 1$ lesions etc.) or may be applied toward a more comprehensive risk model for the individual. However, it needs to be noted that CCI did not represent an independent predictor of disability in this study. Further investigations should be performed prospectively, combining CCI with linear measurements of grey matter (e.g. intercaudate ratio) to better define a role in daily practice.

Acknowledgment We thank Dr. Christian Schindler, local statistician at the Institute of Social and Preventive Medicine at the Swiss Tropical Institute Basel, for providing statistical support toward this study.

\section{References}

1. Anderson VM, Fox NC, Miller DH (2006) Magnetic resonance imaging measures of brain atrophy in multiple sclerosis. J Magn Reson Imaging 23:605-618

2. Barkhof FJ, Elton M, Lindeboom J et al (1998) Functional correlates of callosal atrophy in relapsing-remitting multiple sclerosis patients. A preliminary MRI study. J Neurol 245:153-158

3. Bermel RA, Sharma J, Tjoa CW et al (2003) A semiautomated measure of whole-brain atrophy in multiple sclerosis. J Neurol Sci 208:57-65

4. Chard DT, Griffin CM, Parker GJ et al (2002) Brain atrophy in clinically early relapsing-remitting multiple sclerosis. Brain 125:327-337

5. Chard DT, Griffin CM, Rashid W et al (2004) Progressive grey matter atrophy in clinically early relapsing-remitting multiple sclerosis. Mult Scler 10:387-391

6. Confavreux C, Vukusic S, Adeleine P (2003) Early clinical predictors and progression of irreversible disability in multiple sclerosis: an amnesic process. Brain 126:770-782

7. Dalton CM, Miszkiel KA, O'Connor PW et al (2006) Ventricular enlargement in MS: one-year change at various stages of disease. Neurology 66:693-698

8. De Stefano N, Battaglini M, Smith SM (2007) Measuring brain atrophy in multiple sclerosis. J Neuroimaging 17(Suppl 1):10S$15 \mathrm{~S}$

9. De Stefano N, Matthews PM, Filippi M et al (2003) Evidence of early cortical atrophy in MS: relevance to white matter changes and disability. Neurology 60:1157-1162

10. Evangelou N, Esiri MM, Smith S et al (2000) Quantitative pathological evidence for axonal loss in normal appearing white matter in multiple sclerosis. Ann Neurol 47:391-395

11. Figueira FF, Santos VS, Figueira GM et al (2007) Corpus callosum index: a practical method for long-term follow-up in multiple sclerosis. Arq Neuropsiquiatr 65:931-935

12. Filippi M, Campi A, Colombo B et al (1996) A spinal cord MRI study of benign and secondary progressive multiple sclerosis. J Neurol 243:502-505
13. Filippi M, Mastronardo G, Rocca MA et al (1998) Quantitative volumetric analysis of brain magnetic resonance imaging from patients with multiple sclerosis. J Neurol Sci 158:148-153

14. Filippi M, Paty DW, Kappos L et al (1995) Correlations between changes in disability and T2-weighted brain MRI activity in multiple sclerosis: a follow-up study. Neurology 45:255-260

15. Fox NC, Jenkins R, Leary SM et al (2000) Progressive cerebral atrophy in MS: a serial study using registered, volumetric MRI. Neurology 54:807-812

16. Gasperini C, Horsfield MA, Thorpe JW et al (1996) Macroscopic and microscopic assessments of disease burden by MRI in multiple sclerosis: relationship to clinical parameters. J Magn Reson Imaging 6:580-584

17. Gass A, Barker GJ, Kidd D et al (1994) Correlation of magnetization transfer ratio with clinical disability in multiple sclerosis. Ann Neurol 36:62-67

18. Ge Y, Grossman RI, Udupa JK et al (2001) Brain atrophy in relapsing-remitting multiple sclerosis: fractional volumetric analysis of gray matter and white matter. Radiology 220:606-610

19. Ge Y, Grossman RI, Udupa JK et al (2000) Brain atrophy in relapsing-remitting multiple sclerosis and secondary progressive multiple sclerosis: longitudinal quantitative analysis. Radiology 214:665-670

20. Gean-Marton AD, Vezina LG, Marton KI et al (1991) Abnormal corpus callosum: a sensitive and specific indicator of multiple sclerosis. Radiology 180:215-221

21. Hardmeier M, Wagenpfeil S, Freitag P et al (2003) Atrophy is detectable within a 3-month period in untreated patients with active relapsing remitting multiple sclerosis. Arch Neurol 60:1736-1739

22. Harris JO, Frank JA, Patronas N et al (1991) Serial gadoliniumenhanced magnetic resonance imaging scans in patients with early, relapsing-remitting multiple sclerosis: implications for clinical trials and natural history. Ann Neurol 29:548-555

23. Hines M, Chiu L, McAdams LA et al (1992) Cognition and the corpus callosum: verbal fluency, visuospatial ability, and language lateralization related to midsagittal surface areas of callosal subregions. Behav Neurosci 106:3-14

24. Horakova D, Cox JL, Havrdova E et al (2008) Evolution of different MRI measures in patients with active relapsing-remitting multiple sclerosis over 2 and 5 years: a case-control study. J Neurol Neurosurg Psychiatry 79:407-414

25. Jones CK, Riddehough A, Li DKB et al (2001) MRI cerebral atrophy in relapsing-remitting MS: Results from the PRISMS Trial. Neurology 56:A379

26. Kalkers NF, Ameziane N, Bot JC et al (2002) Longitudinal brain volume measurement in multiple sclerosis: rate of brain atrophy is independent of the disease subtype. Arch Neurol 59:1572-1576

27. Kassubek J, Tumani H, Ecker D et al (2003) Age-related brain parenchymal fraction is significantly decreased in young multiple sclerosis patients: a quantitative MRI study. Neuroreport 14:427430

28. Kidd D, Thorpe JW, Thompson AJ et al (1993) Spinal cord MRI using multi-array coils and fast spin echo. II. Findings in multiple sclerosis. Neurology 43:2632-2637

29. Liu C, Edwards S, Gong Q et al (1999) Three dimensional MRI estimates of brain and spinal cord atrophy in multiple sclerosis. J Neurol Neurosurg Psychiatry 66:323-330

30. Losseff NA, Wang L, Lai HM et al (1996) Progressive cerebral atrophy in multiple sclerosis. A serial MRI study. Brain 119(Pt 6): 2009-2019

31. Lublin FD, Reingold SC (1996) Defining the clinical course of multiple sclerosis: results of an international survey. National Multiple Sclerosis Society (USA) Advisory Committee on Clinical Trials of New Agents in Multiple Sclerosis. Neurology 46:907-911 
32. Martola J, Stawiarz L, Fredrikson S et al (2007) Progression of non-age-related callosal brain atrophy in multiple sclerosis: a 9-year longitudinal MRI study representing four decades of disease development. J Neurol Neurosurg Psychiatry 78:375-380

33. Miller DH, Albert PS, Barkhof F et al (1996) Guidelines for the use of magnetic resonance techniques in monitoring the treatment of multiple sclerosis. US National MS Society Task Force. Ann Neurol 39:6-16

34. Miller DH, Barkhof F, Frank JA et al (2002) Measurement of atrophy in multiple sclerosis: pathological basis, methodological aspects and clinical relevance. Brain 125:1676-1695

35. Miller DH, Grossman RI, Reingold SC et al (1998) The role of magnetic resonance techniques in understanding and managing multiple sclerosis. Brain 121(Pt 1):3-24

36. Myhr KM, Riise T, Vedeler C et al (2001) Disability and prognosis in multiple sclerosis: demographic and clinical variables important for the ability to walk and awarding of disability pension. Mult Scler 7:59-65

37. Nijeholt GJ, van Walderveen MA, Castelijns JA et al (1998) Brain and spinal cord abnormalities in multiple sclerosis. Correlation between MRI parameters, clinical subtypes and symptoms. Brain 121(Pt 4):687-697

38. Pelletier J, Suchet L, Witjas T et al (2001) A longitudinal study of callosal atrophy and interhemispheric dysfunction in relapsingremitting multiple sclerosis. Arch Neurol 58:105-111

39. Polman CH, Reingold SC, Edan G et al (2005) Diagnostic criteria for multiple sclerosis: 2005 revisions to the "McDonald Criteria”. Ann Neurol 58:840-846

40. Quarantelli M, Ciarmiello A, Morra VB et al (2003) Brain tissue volume changes in relapsing-remitting multiple sclerosis: correlation with lesion load. Neuroimage 18:360-366

41. Rudick RA, Fisher E, Lee JC et al (1999) Use of the brain parenchymal fraction to measure whole brain atrophy in relapsing-remitting MS. Multiple Sclerosis Collaborative Research Group. Neurology 53:1698-1704

42. Runmarker B, Andersen O (1993) Prognostic factors in a multiple sclerosis incidence cohort with twenty-five years of follow-up. Brain 116(Pt 1):117-134
43. Sanfilipo MP, Benedict RH, Sharma J et al (2005) The relationship between whole brain volume and disability in multiple sclerosis: a comparison of normalized gray versus white matter with misclassification correction. Neuroimage 26:1068-1077

44. Sharma J, Sanfilipo MP, Benedict RH et al (2004) Whole-brain atrophy in multiple sclerosis measured by automated versus semiautomated MR imaging segmentation. AJNR Am J Neuroradiol 25:985-996

45. Simon JH, Holtas SL, Schiffer RB et al (1986) Corpus callosum and subcallosal-periventricular lesions in multiple sclerosis: detection with MR. Radiology 160:363-367

46. Tedeschi G, Lavorgna L, Russo P et al (2005) Brain atrophy and lesion load in a large population of patients with multiple sclerosis. Neurology 65:280-285

47. Tiberio M, Chard DT, Altmann DR et al (2005) Gray and white matter volume changes in early RRMS: a 2-year longitudinal study. Neurology 64:1001-1007

48. Truyen L, van Waesberghe JH, van Walderveen MA et al (1996) Accumulation of hypointense lesions ("black holes") on T1 spinecho MRI correlates with disease progression in multiple sclerosis. Neurology 47:1469-1476

49. Valsasina P, Benedetti B, Rovaris M et al (2005) Evidence for progressive gray matter loss in patients with relapsing-remitting MS. Neurology 65:1126-1128

50. van Walderveen MA, Barkhof F, Hommes OR et al (1995) Correlating MRI and clinical disease activity in multiple sclerosis: relevance of hypointense lesions on short-TR/short-TE (T1-weighted) spin-echo images. Neurology 45:1684-1690

51. Vrenken H, Geurts JJ, Knol DL et al (2006) Whole-brain T1 mapping in multiple sclerosis: global changes of normalappearing gray and white matter. Radiology 240:811-820

52. Zivadinov R, Reder AT, Filippi M et al (2008) Mechanisms of action of disease-modifying agents and brain volume changes in multiple sclerosis. Neurology 71:136-144 